\title{
Concept and Design of the Intellwheels Platform for Developing Intelligent Wheelchairs
}

\author{
Rodrigo A. M. Braga ${ }^{1,2}$, Marcelo Petry ${ }^{2}$, Antonio Paulo Moreira ${ }^{2}$, Luis Paulo Reis ${ }^{1,2}$ \\ ${ }^{1}$ LIACC - Artificial Intelligence and Computer Science Lab., \\ ${ }^{2}$ FEUP-DEI - Faculty of Engineering of the University of Porto, Informatics Engineering \\ Department, Rua Dr. Roberto Frias, s/n 4200-465, Porto, PORTUGAL \\ rodrigo.braga@fe.up.pt, marcelo.petry@gmail.com, amoreira@fe.up.pt, lpreis@ fe.up.pt,
}

\begin{abstract}
Many people with severe disabilities find it difficult or even impossible to use traditional powered wheelchairs independently by manually controlling these electrical devices. Intelligent wheelchairs are a very good solution to assist severely handicapped people who are unable to operate classical electrical wheelchair by themselves in their daily activities. This paper describes a development platform for intelligent wheelchairs called IntellWheels. The intelligent system developed may be added to commercial powered wheelchairs with minimal modifications in a very straightforward manner. The paper describes the concept and design of the platform, including the hardware and software, multimodal input interface and the intelligent wheelchair prototype developed to validate the approach. Preliminary results concerning automatic movement of the IntellWheels prototype are also described showing the autonomous movement capabilities of the prototype.
\end{abstract}

Keywords: Intelligent Wheelchair, Intelligent Robotics, Human-Robot Interfaces.

\section{Introduction}

Wheelchairs are important locomotion devices for handicapped and senior people. With the increase in the number of senior citizens and the increment of people bearing physical deficiencies in the social activities, there is a growing demand for safer and more comfortable Intelligent Wheelchairs (IW) for practical applications. The main functions of IWs are [9][3][4]:

- Interaction with the user, including hand based control (such as, joystick, keyboard, mouse, touch screen); voice based control; vision based control and other sensor based control (such as pressure sensors);

- Autonomous navigation (with safety, flexibility and robust obstacle avoidance);

- Communication with other devices (like automatic doors and other Wheelchairs). 
This paper discusses the concept and the design of a development platform for intelligent wheelchairs. The project, called IntellWheels, is composed of a control software, simulator/supervisor and a real prototype of an intelligent wheelchair. In the study, shared control and high-level planning algorithms applied in an IW operating in a hospital environment were developed and tested. A shared control algorithm was tested, allowing the IW to automatically avoid dangerous situations. Also, typical algorithms used in most intelligent robotics applications were applied in the control of the IW and simulated in a hospital scenario. Blended with the control, a motion planner was developed capable of generating the behavior/path commands according to an a-priori created map of the world. This motion planner is capable of instructing the low-level motion controller module to achieve the high-level commands desired by the user [12].

The rest of the paper is subdivided as following different sections: Section 2 presents some related work; section 3 explains the hardware design of our development platform; section 4 and 5 contain a complete description of the software design and control system; section 6 provides experimental tests and result discussion and section 7 presents the final conclusions and points out some future research topics.

\section{Related Work}

This section presents a brief to the state of the art about Intelligent Wheelchairs with focus on the most recent research work on the development of IW prototypes.

In recent years, many intelligent wheelchairs have been developed and a large number of scientific projects were started in the area [19]. Only in the year of 2006 more than 30 publications in IEEE, about IW, may be found.

One of the first concept projects of an autonomous wheelchair for handicapped physicists was proposed by Madarasz [13]. He presented a wheelchair equipped with a microcomputer, a digital camera, and ultrasound scanner. His objective was to develop a vehicle capable to operate without human intervention in populated environments with little or no collision with the objects or people contained in it.

Hoyer and Holper [7] presented an architecture of a modular control for a omnidirectional wheelchair. NavChair is described in [20], [2], [11] and has some interesting characteristic such as wall following, automatic obstacle avoidance, and doorways passing capabilities.

Miller and Slak [14], [15] projected the Tin Man I system, initially with three ways of operation: human guided with obstacle avoidance, move forward along a heading, move to a point (x, y). Afterwards, the project Tin Man I evolved, resulting in several new functions, extended in the Tin Man II, by including new capabilities such as: backup, backtracking, wall following, doorway passing, and docking.

Wellman [22] proposed a hybrid wheelchair which is equipped with two legs additionally to the four wheels, which enable the wheelchair to climb over steps and move through rough terrain.

Some projects presented solutions for people with tetraplegia, by using the recognition of facial expressions as the main input to guide the wheelchair [8], [16], 
[1]. Another method of control is by using the user "thoughts". This technology typically uses sensors that measure the electromagnetic waves of the brain [10], [17].

ACCoMo [6] is a prototype of an IW that allows for safe movement in indoor environments for the handicapped physicists. ACCoMo is an agent based prototype with simple autonomous, cooperative and collaborative behaviors.

Although several prototypes have been developed and different approaches have been proposed for IWs, there is not, at the moment, a proposal for a platform enabling easy development of Intelligent Wheelchairs using common electric powered wheelchairs with minor modifications.

\section{Hardware Design}

The hardware architecture of IntellWheels prototype is shown in Figure 1. The IntellWheels_chair1 is based on a commercial electrical wheelchair model Powertec, manufactured by Sunrise in England [21]. The Powertec wheelchair has following features: Two differentially driven rear wheels; Two passive castor front; Two 12V batteries (45Ah); Traditional Joystick; Power Module.

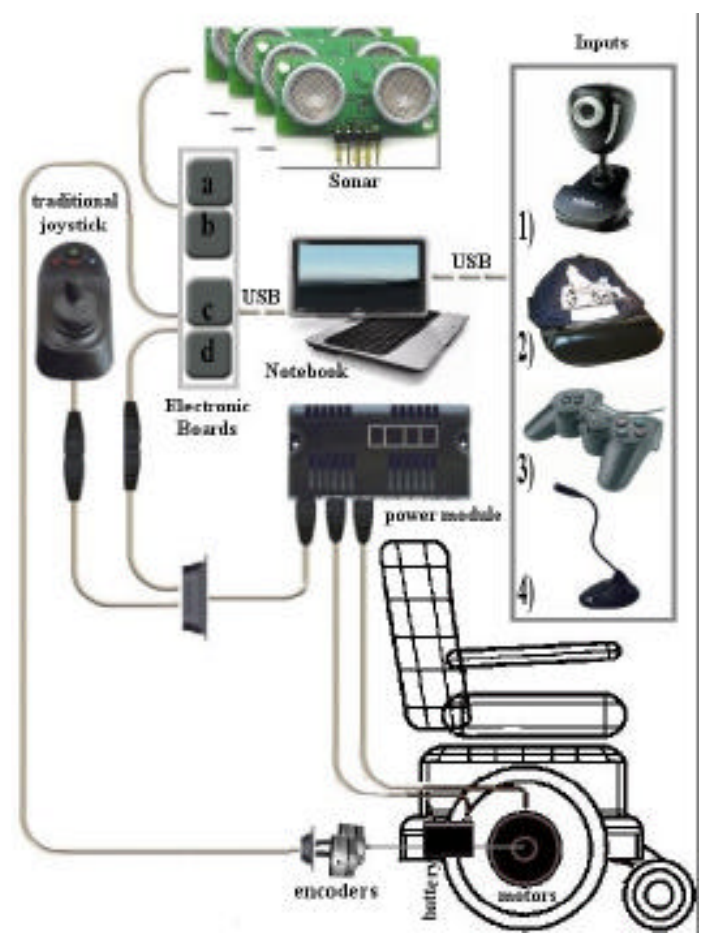

Fig. 1. Hardware Architecture of IntellWheels. 
The IntellWheels hardware parts are divided in three functional blocks: user inputs, IW sensor, hardware devices. These blocks are depicted in Figure 2.

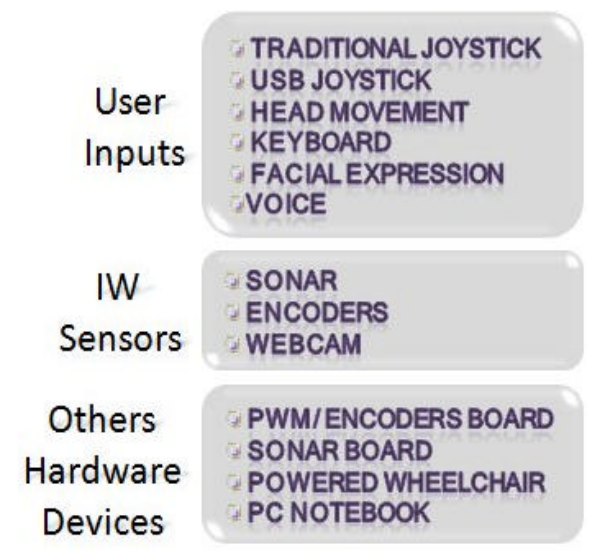

Fig. 2. Hardware Functional Blocks.

\subsection{User Inputs}

To enable people with different kinds of disabilities to drive the intelligent wheelchair, this project has incorporated several sorts of user inputs. The idea is to give options to the patients, and let them choose what control is more comfortable and safer. Besides that, multiple inputs makes possible the IntellWheels integration with an intelligent input decision control, which is responsible to cancel inputs in the case its perception recognizes conflicts, noise and danger. For example, in a noisy environment, voice recognition would have low rate or even would be canceled. The inputs implemented go from traditional joysticks until head movement control, and are explained bellow:

- Traditional Joysticks. These inputs present in ordinary wheelchairs are a robust way to drive a wheelchair. However they may not be accessible to paraplegic or cerebral palsy people. They are present in the prototype due to its simplicity;

- USB Joystick. The USB joysticks are a little bit more sophisticated than traditional joysticks. This game joystick has many configurable buttons that makes the navigation easier;

- Head Movement. This input device is mounted in a cap making it possible to drive the wheelchair just with head movement (Figure 3);

- Keyboard. This device enables that the wheelchair control can be made just pushing some keys in the keyboard;

- Facial Expressions. By using a simple webcam, present in most of the notebooks, this software recognize simple facial expressions of the patient, 
using them as inputs to execute since basic commands (like: go forward, right and left) to high level command (like: go to nursery, go to bedroom);

- Voice. Using commercial software of voice recognition we developed the necessary conditions and applications to command the wheelchair using voice as an input.

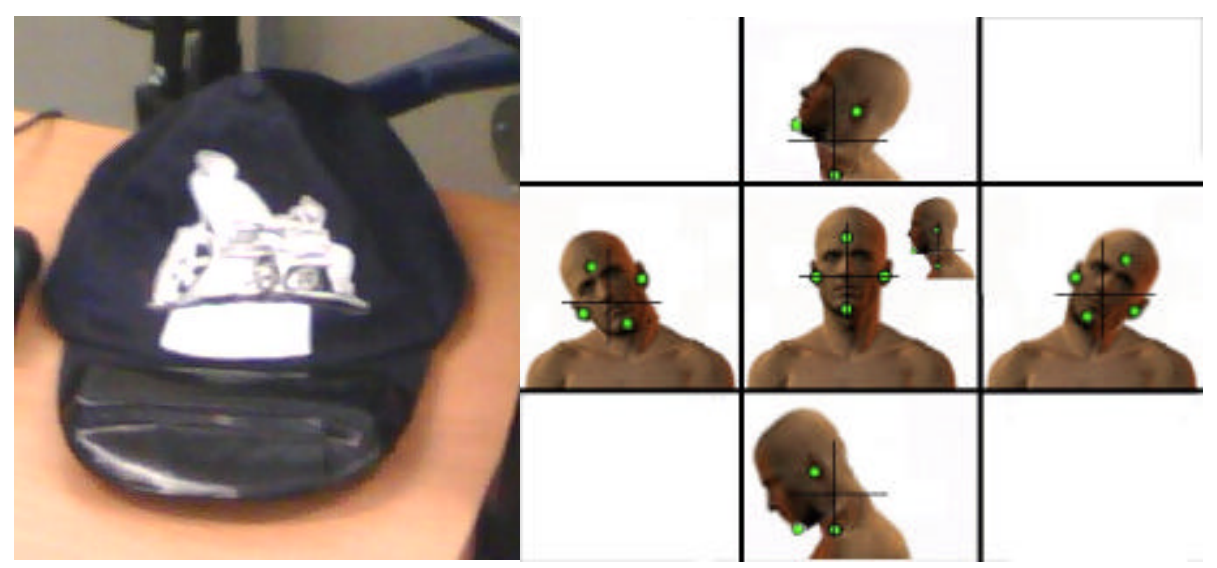

Fig. 3. Sensor for head movement detect

The use of a vast set of input options enables the prototype to be easily controlled by patients suffering from distinct disabilities. All the inputs are integrated in a very flexible multimodal interface [4].

\subsection{Sensors}

The purpose of this project is to develop a really intelligent wheelchair capable of performing semi-autonomous actions and having a high-level interaction with the user. However, the distinction between an IW and a robotic wheelchair is not just semantic. It means that we want to keep the wheelchair appearance, reducing the visual impact that the sensors mounted on the device produce but, at the same time, increasing the wheelchair regular functionalities. This statement limits the number and the kind of sensors we are able to use due to size, appearance and assembly constraints. To compose the intelligent wheelchair ten sonar sensors were mounted (giving the ability to avoid obstacles, follow walls and perceive unevenness in the ground), two encoders were assembled on the wheels (providing the tools to measure distance, speed, position) and two webcams were placed (one directed to the ground, capable to read ground marks and refine the odometry and the other directed to the patient head to detect facial expressions). 


\subsection{Hardware Devices}

The hardware devices block is composed of (Figure 4):

- 2 sonar board (Electronic board ' $a$ ' and ' $b$ ' illustrated in the Figure 1 and Figure 2), the function ' $a$ ' and ' $b$ ' boards are receiving information of the ten sonars and sending it to the PC;

- 2 PWM/Encoders board (Electronic board 'c' and 'd'), these boards have speed control function, as well as sending to the PC the displacement information to enable the odometry;

- Commercial electrical wheelchair and commercial notebook.

The core of IntellWheels prototype is a PC notebook (HP Pavilion tx1270EP,AMD Turion 64 X2 TI60), although other notebooks could be used without any loss of capabilities.

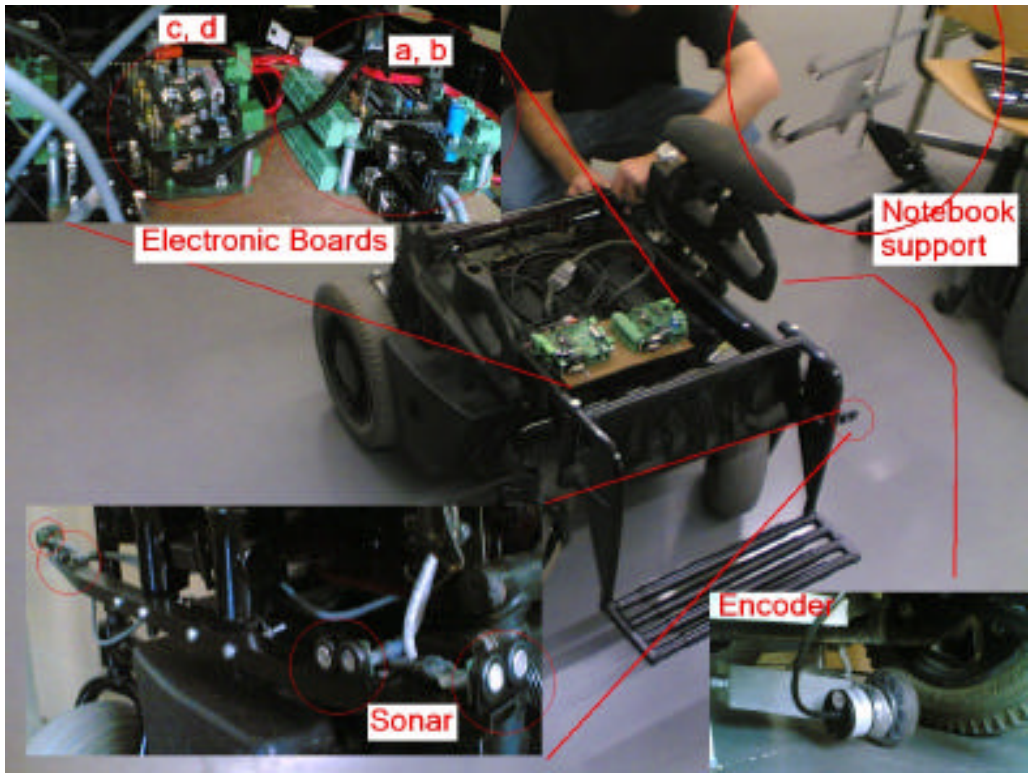

Fig. 4. Devices installed in IW.

\section{Software Design}

The software in this project is composed by a modular system, where each group of tasks interacts with the main application. Figure 5 shows the specific functions and the software responsible to convert data from hardware to be used in the control level. 


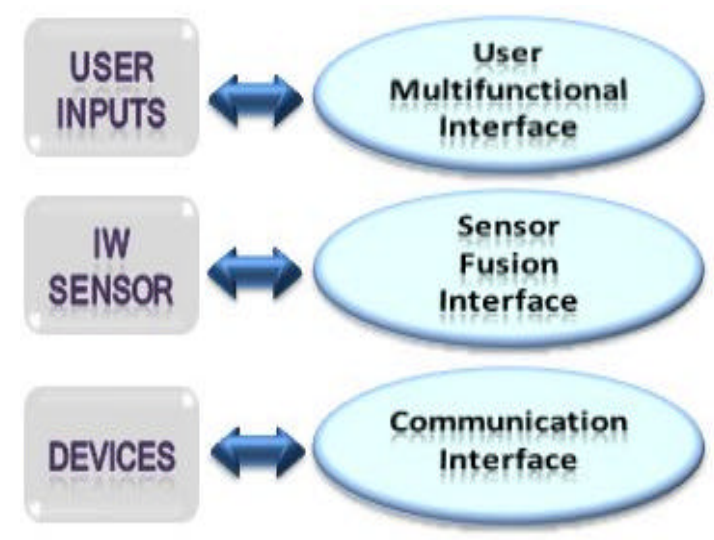

Fig. 5. Intelligent Wheelchair Software.

The main application has the function to gather all information from the system, communicate with the control module and set the appropriate output. Moreover, it has other different functions depending on what is the control interface mode set:

- Real. If this option is selected, all data comes from the real world. The main application collects real information from sensors through the Sensor User Interface, calculate the output through the Control module and send these parameters to the PWM Boards;

- Simulated. In this mode, the system works just with virtual information and is used for two purposes: generate the same behavior of a real wheelchair and to test control routines. The main application collects virtual information directly from the simulator, calculates the output through the Control module and send back these parameters to the simulator;

- Augmented Reality. This mode creates an interaction between real and virtual objects, changing a real wheelchair's path to avoid collision with a virtual wall (or with a virtual wheelchair) for example. The objective of the augmented reality is to test the interaction between wheelchairs and environment, reducing its costs once major agents can be simulated. The main application collects real information from the sensors through the Sensor User Interface and mix it with virtual information collected from the simulator, calculate the output through the Control module and send the parameters to the simulator and the PWM Boards.

Due to its capabilities to interact with real, simulated and augmented reality worlds we call this whole system: IW Platform (Figure 6). In other words, the Platform is the fusion of the Simulator, Software modules, Real Wheelchair and Hardware Devices, to test, preview, understand and simulate the behaviour of Intelligent Wheelchairs. 


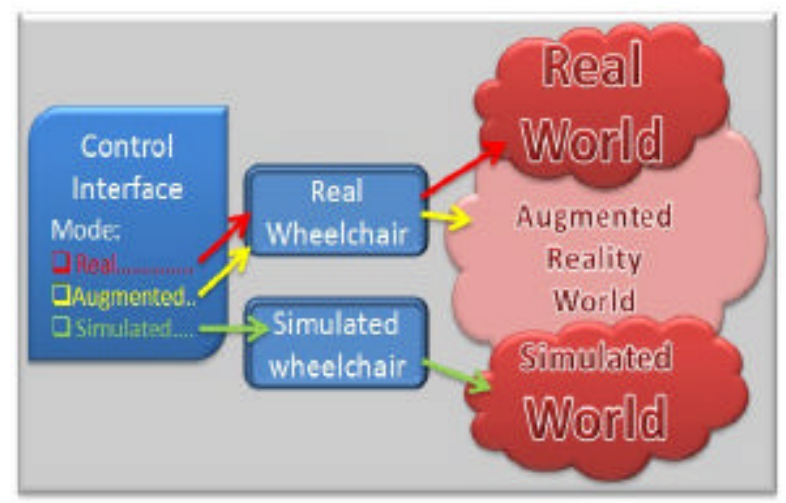

Fig. 6. Intelligent Wheelchair Platform Architecture.

\section{Control Architecture}

IntellWheels has a multi-level control architecture subdivided in three levels: a basic control level, a tactical level and strategy level, as shown in Figure 7.

As focus is primarily testing the Platform, classic algorithms were chosen to validate the system, some other issues are left outside the scope of this paper, such as analyses of its limitations and its performance. High Level strategy planning is responsible to create a sequence of high level actions needed to achieve the global goal. Actually the algorithm implemented to fulfill this task is based on the STRIPS planning algorithm [5].

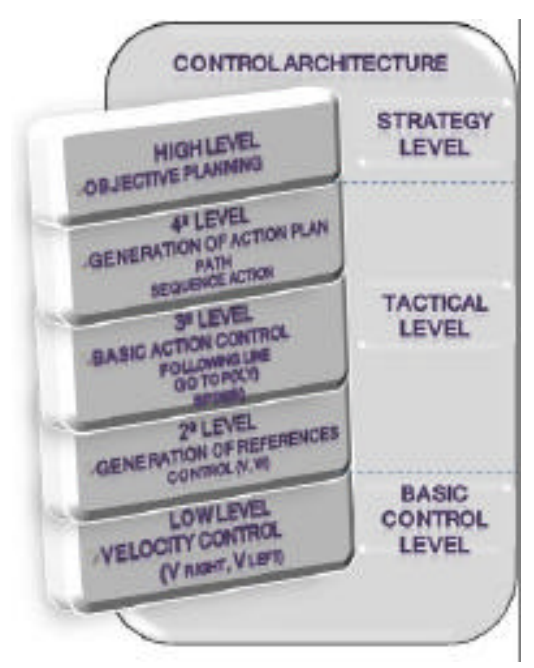

Fig. 7. Control architecture. 
In the Generation of Action Plans, we order the system to generate a sequence of basic actions aiming to satisfy the objectives proposed previously. To find a path from a given initial node to a given goal node the system has a simple $A^{*}$ Algorithm implemented [18]. Once the path is calculated, it is subdivided into basic forms (lines, circles, points) that are afterwards followed by the wheelchair.

Each basic form represents a basic action to be executed on time in the Basic Action Control module. Following that the system executes the Generation of References, which is responsible to estimate the wheelchair linear and angular speeds, putting the wheelchair into motion.

The lowest level of control is designed through a Digital PID implemented in the PWM/Encoders Boards. References from Basic Action are transferred by serial communication to the boards and then contrasted with real time data to control the speed.

Probabilistic robotics is a new and growing area in robotics, concerned with perception and control in the face of uncertainty. Building on the field of mathematical statistics, probabilistic robotics endows robots with a new level of robustness in real-world situations [23]. In this work was utilized probabilistic odometry motion model for an active localization system. When the uncertainty (variance) in the IW position, given by the odometry, grows up, the path planning changes the trajectory in a way that the IW goes near a landmark and resets its localization. This probabilistic model is also useful to decide how many landmarks are needed and where to put them.

\section{Experiments and Results}

This section presents the prototype implementation and a simple experiment and the results achieved. Figure 8 shows the mechanical structure and the hardware implementation. As we can see, the prototype is a commercial electric powered wheelchair with minimal modifications.

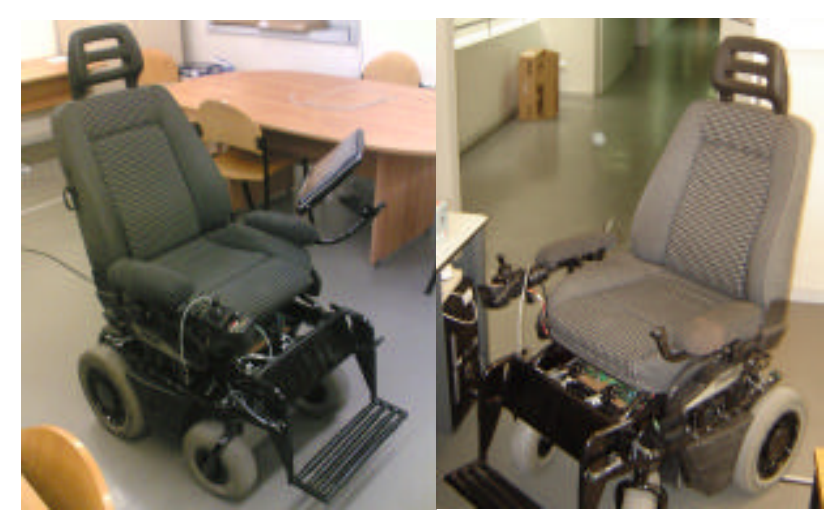

Fig. 8. IntellWheels_Chair1 Prototype. 
User interface software was designed to be as friendly as possible and it is shown in Figure 9. In the main window it contains a webcam window, which is used to recognize landmarks, and the results of its localization. A schematic figure with the position of the sonar mounted in the wheelchair easily shows the distance to nearby objects. By the side, we have a panel with the information provided by the odometry. The application still displays a scrollbar indicating the speed of each wheel as well as buttons to choose the operation mode.

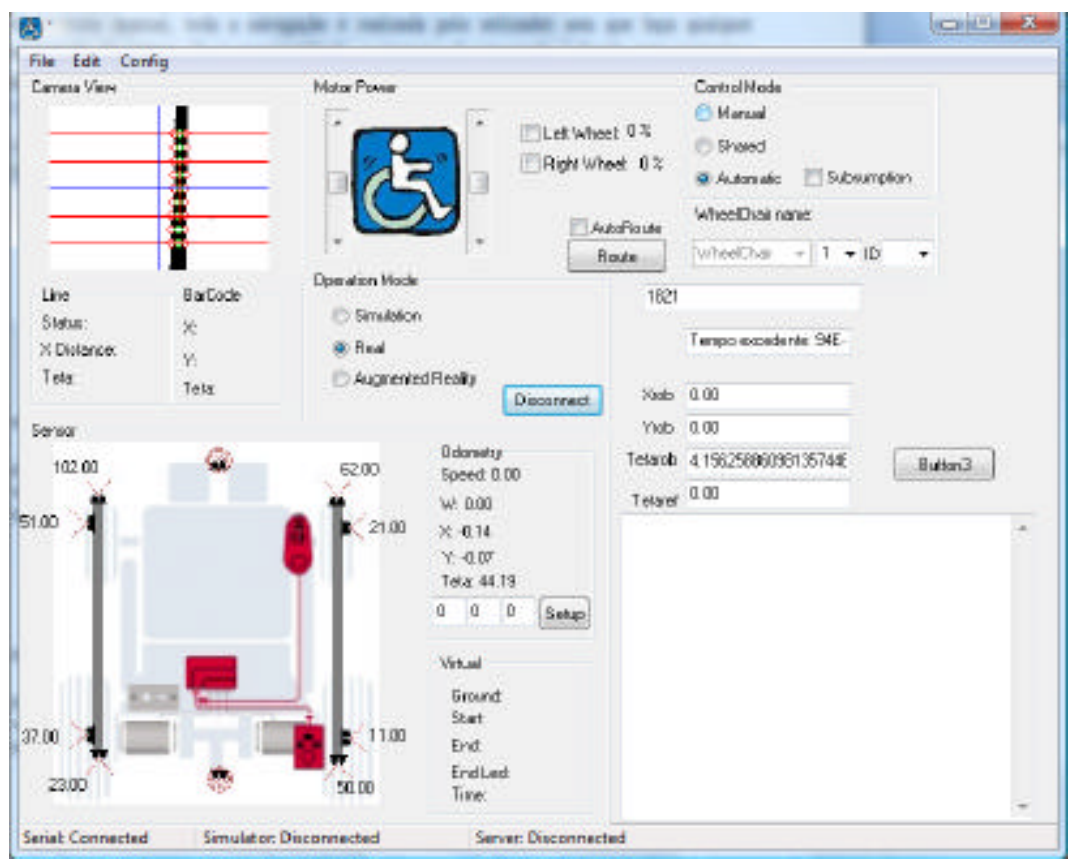

Fig. 9. Interface Control of IntellWheels.

Some basic tests were performed to validate odometry, and consisted in moving the wheelchair around a rectangle path (Figure 10), starting and stopping in the same point. In Figure 11 the results of an automatic test (red line) and a manual test (blue line) are presented. In the manual test the user had all control of the wheelchair.

In the automatic mode the final point of the path of the wheelchair presented a little displacement regarding its start point (physical mark point on the ground). As this error is not displayed in the odometry graph, a Manual test was executed to evaluate the results. In this test, the user drove the wheelchair following the same path, but at this time stopping in the same physical mark point on the ground. Results of this test presented the error displayed in the odometry graph. 


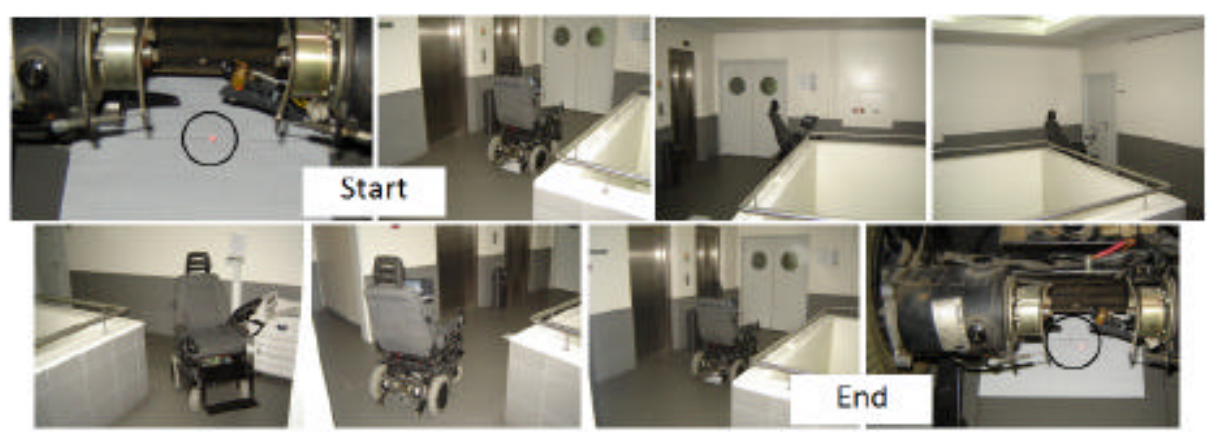

Fig. 10. Rectangle path being followed by the Wheelchair in the experiments.

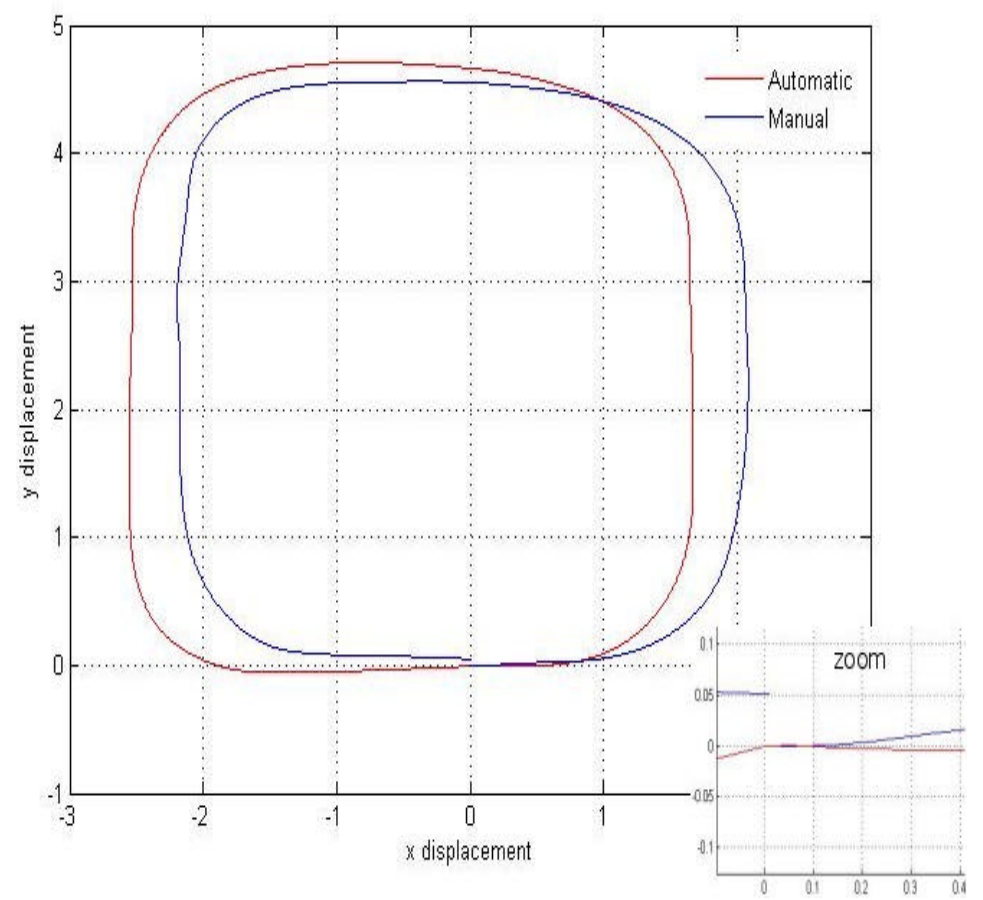

Fig. 10. Path Followed by Wheelchair in the Tests.

The error presented is admissible since it is just $5 \mathrm{~cm}$ in a total amount of $1500 \mathrm{~cm}$ of displacement, and can be explained by the integration of the odometry systematic error.

Figure 12 and Figure 14 shows the results of distinct tests of displacement in straight line. The objective was evaluate odometry dispersion error for different distances. In this test was valued displacement of 5, 10 and 15 meters, with approximately 20 samples for each path. 


\section{$7 \quad$ Discussion and Conclusions}

This paper presented the design and implementation of a development platform for Intelligent Wheelchairs called IntellWheels. This platform facilitates the development and test of new methodologies and techniques concerning Intelligent Wheelchairs. We believe that the new techniques developed may bring to the wheelchairs real capacities of intelligent action planning, autonomous navigation, and mechanisms to allow the execution, in a semi-autonomous way, of the user desires, expressed in a high-level language of command.

Future research will aim the test and validation for the other sensors mounted in the wheelchair prototype. Moreover, like in many other systems, cooperative and collaborative behaviours are desired to be present in the IW and need to be incorporated in the platform. Another important improvement to be pursued includes a comparative study of the classic implemented algorithms and the new proposal to solve these issues.

The platform will allow that real and virtual IWs interact with each other. These interactions make possible high complexity tests with a substantial number of devices and wheelchairs, representing a reduction in the project costs, once there is not the necessity to build a large number of real IW.

\section{Acknowledgments}

The first author would like to thank for CAPES for doctoral course financing. This work was partially supported by the project ACORD - Adaptative Coordination of Robotic Teams - FCT/PTDC/EIA/70695/2006.

\section{References}

1. Adachi,Y., Kuno, Y., Shimada, N., Shirai, Y.: Intelligent wheelchair using visual information on human faces. International Conference in Intelligent Robots and Systems. Oct. Vol. 1. pp. 354-359 (1998)

2. Bell, D.A., Borenstein, J., Levine, S.P., Koren, Y., and Jaros, L.: An assistive navigation system for wheelchairs based upon mobile robot obstacle avoidance. IEEE Conf. on Robotics and Automation (1994)

3. Faria, P. M., Braga, R A. M., Valgôde, E., Reis L. P.: Platform to Drive an Intelligent Wheelchair using Facial Expressions. Proceedings of the $9^{\text {th }}$ International Conference on Enterprise Information Systems - Human-Computer Interaction (ICEIS 2007). pp. 164-169. Funchal Madeira, Portugal. June 12-16. ISBN: 978-972-8865-92-4 (2007)

4. Faria, P. M., Braga, R. A. M., Valgôde, E., Reis, L. P. Interface Framework to Drive an Intelligent Wheelchair Using Facial Expressions. IEEE International Symposium on Industrial Electronics. 4-7 de June de 2007, pp. 1791-1796, ISBN: 1-4244-0755-9 (2007)

5. Fikes, R., Nilsson, N. J.: STRIPS: A New Approach to the Application of Theorem Proving to Problem Solving. IJCAI 1971. pp.608-620 (1971) 
6. Hamagami, T., Hirata, H.: Development of intelligent wheelchair acquiring autonomous, cooperative, and collaborative behavior. IEEE International Conference on Systems, Man and Cybernetics. Oct. Vol. 4. pp. 3525 - 3530 (2004)

7. Hoyer, H. and Hölper, R.: Open control architecture for an intelligent omnidirectional wheelchair. Proc.1st TIDE Congress, Brussels. pp. 93-97 (1993)

8. Jia, P. and Hu, H.: "Head Gesture based Control of an Intelligent Wheelchair", CACSUK 11th Annual Conference of the Chinese Automation and Computing Society in the UK, Sheffield, UK, Sept. 10 (2005)

9. Jia, P., Hu, H., Lu, T. and Yuan, K., Head Gesture Recognition for Hands-free Control of an Intelligent Wheelchair. Journal of Industrial Robot (2006)

10. Lakany, H.: Steering a wheelchair by thought. The IEEE International Workshop on Intelligent Environments. June. pp. 199 - 202 (2005)

11. Levine, S.P., Bell, D.A., Jaros, L.A., Simpson, R.C., Koren, Y., Borenstein, J.: The NavChair assistive wheelchair navigation system. IEEE Transactions on Rehabilitation Engineering pp. 443-451 (1997)

12. Luo, R. C., Chen, T. M. and Lin, M. H.: Automatic Guided Intelligent Wheelchair System Using Hierarchical Grey-Fuzzy Motion Decision-Making Algorithms, Proc. of IEEVRSJ'99 - International Conference on Intelligent Robots and Systems (1999)

13. Madarasz, R.L., Heiny, L.C., Cromp, R.F. and Mazur, N.M.: The design of an autonomous vehicle for the disabled, IEEE J. Robotics and Automat. September, pp. $117-126$ (1986)

14. Miller, D. and Slack, M.: Design and testing of a low-cost robotic wheelchair prototype. Autonomous Robots. Vol. 2, pp. 77-88 (1995)

15. Miller, D. Assistive Robotics: An Overview. Assistive Technology and AI. pp. 126-136 (1998)

16. Pei Chi Ng; DeSilva, L.C.: Head gestures recognition. Proceedings of the International Conference on Image Processing. Oct. Vol. 3. pp. 266-269. ISBN: 0-7803-6725-1 (2001)

17. Rebsamen, B., Burdet, E., Guan, C., Zhang, H., Teo, C. L., Zeng, Q., Laugier, C., Ang Jr., M. H.: Controlling a Wheelchair Indoors Using Thought. IEEE Intelligent Systems and Their Applications. Vol. 22, pp. 18 - 24 (2007)

18. Shapiro S.C., 2000. Encyclopedia of Artificial Intelligence. Wiley-Interscience. May.

19. Simpson, R. C.: Smart wheelchairs: A literature review. Journal of Rehabilitation Research \& Development. August. Vol. 42. pp. 423-436 (2005)

20. Simpson, R. et al.: NavChair: An Assistive Wheelchair Navigation System with Automatic Adaptation. [ed.] Mittal et al. Assistive Technology and AI. pp. 235-255 (1998)

21. Sunrise, Sunrise Medical manufacturers www.sunrisemedical.co.uk (2007)

22. Wellman, P., Krovi, V. and Kumar, V.: An adaptive mobility system for the disabled. Proceedings of the IEEE International Conference on Robotics and Automation (1994)

23. Thrun, S., Burgard, W. and Fox, D.: Probabilistic Robotics. The MIT Press. Cambridge. pp. 117-145. ISBN 0-262-20162-3 (2005) 